\title{
Large Atoms in the Magnetic Field of a Neutron Star
}

\author{
Elliott H. Lieb
}

\begin{abstract}
.
The asymptotics of the ground state energy of large atoms as $Z \rightarrow \infty$ is given exactly by Thomas-Fermi theory. The introduction of a large magnetic field, $B$, changes the situation. If we set $B=c Z^{p}$ then, as $Z \rightarrow \infty$, there are 5 regions: $p<4 / 3, p=4 / 3,4 / 3<p<3$, $p=3, p>3$. The first three are described exactly by a modified TF theory. The fifth is describable exactly by a one-dimensional Hartree like theory. The fourth is describable exactly by a novel density matrix theory. A surprising conclusion is that although the magnetic field has a profound effect on the atomic energy in regions $2,3,4$ and 5 , the atom remains spherical (to leading order) in regions 2 and 3.
\end{abstract}

\section{$\S 1$. Introduction}

In this talk I shall discuss the effect on matter, specifically the ground state of atoms, of a very strong magnetic field. Results obtained in collaboration with J.P. Solovej and J. Yngvason will be summarized and details will appear elsewhere [LSY]. The physical motivation for studying extremely strong magnetic fields of the order of $10^{12}-10^{13}$ Gauss is that they are supposed to exist on the surface of neutron stars. This study was essentially begun in the early 70's with the work of Kadomtsev [K], Ruderman [R] and Mueller, Rau and Spruch [MRS]; see [FGP] and [FGPY] for further references. The argument given to explain these strong fields is that in the collapse, resulting in the neutron star, the magnetic field lines are trapped and thus become very dense. The structure of matter in strong magnetic fields is, therefore, a question of considerable interest in astrophysics. Mathematically, the problem turns out to involve an interesting exercise in semiclassical analysis.

Received September 7, 1992.

(C)1992 in image and content by the author. Reproduction of this article in its entirety by any means is permitted. 
We use units in which $e=\hbar=2 m_{e}=1$ and $c=c \hbar e^{-2}=\alpha^{-1} \approx$ 137. The natural unit of length is $\hbar^{2} / 2 m_{e} e^{2}$, i.e., half the Bohr radius. The natural unit of magnetic field strength that we shall use is $\left(2 m_{e}\right)^{2} e^{3} c / \hbar^{3}=9.4 \times 10^{9}$ Gauss. This is the field for which the magnetic length $\sqrt{c \hbar / e B}$ equals half the Bohr radius. Thus, in our units, $B \approx 10^{2}-10^{3}$ for some neutron stars.

The atomic nucleus of principal interest on the surface of a neutron star is presumably iron with $Z=26$. This number is large and hence it is sensible to ask (rigorously) about the limit of the ground state energy of an atom as $Z \rightarrow \infty$. We shall calculate this limit exactly; its application to $Z=26$ instead of $Z=\infty$ will entail some errors - for which we can give bounds.

\section{§2. Main results}

To give the quantum mechanical energy of a charged spin- $\frac{1}{2}$ particle in a magnetic field $\mathbf{B}$, we have to make a choice of vector potential $\mathbf{A}(x)$, satisfying $\mathbf{B}=\nabla \times \mathbf{A}$. The energy is then given by the Pauli Hamiltonian

$$
H_{\mathbf{A}}=((\mathbf{p}-\mathbf{A}(x)) \cdot \sigma)^{2} .
$$

Here $\mathbf{p}=-i \nabla$ and $\sigma=\left(\sigma_{1}, \sigma_{2}, \sigma_{3}\right)$, are the Pauli matrices. We can also write $H_{\mathbf{A}}=(\mathbf{p}-\mathbf{A})^{2}-\mathbf{B} \cdot \sigma$. We shall here concentrate on the case where $\mathbf{B}$ is constant, say $\mathbf{B}=(0,0, B)$, with $B \geq 0$. We choose $\mathbf{A}=\frac{1}{2} \mathbf{B} \times x$.

The Hamiltonian describing an atom with $N$ electrons and nuclear charge $Z$ (with fixed nucleus) in a constant magnetic field $\mathbf{B}$ is

$$
H_{N}=\sum_{i=1}^{N}\left(H_{\mathbf{A}}^{(i)}-Z\left|x_{i}\right|^{-1}\right)+\sum_{1 \leq i<j \leq N}\left|x_{i}-x_{j}\right|^{-1} .
$$

$H_{N}$ acts on the Hilbert space $\mathcal{H}_{N}=\bigwedge^{N} L^{2}\left(\mathbf{R}^{3} ; \mathbf{C}^{2}\right)$ of antisymmetric (i.e., fermionic) spinor-valued functions. We are interested in $E(N, B, Z)=$ inf $\operatorname{spec}_{\mathcal{H}_{N}} H_{N}$, the ground state energy of $H_{N}$.

We want to let $B$ and $Z$ go to infinity. It is surprising, but true, that there are five different regimes in $B$ and $Z$, depending on the relative magnitudes of $B$ and $Z$. In the following $\rho(x)$ is the electron density in the ground state $\psi$ :

$$
\rho(x)=N \int\left\|\psi\left(x, x_{2}, \ldots, x_{N}\right)\right\|^{2} d^{3} x_{2} \ldots d^{3} x_{N} .
$$


The five regions are the following.

1) $B \ll Z^{4 / 3}, Z$ large:

The effect of the magnetic field is negligible. Standard Thomas-Fermi (TF) theory is exact as $Z \rightarrow \infty$, and therefore the electron density is spherical to leading order.

2) $B \sim Z^{4 / 3}, Z$ large:

The magnetic field becomes important but the density is still almost spherical and stable atoms are almost neutral (see [Y]). A modified TF theory (depending on the constant $B / Z^{4 / 3}$ ), in which the energy, as in standard TF theory, is approximated by a functional of the density $\rho$ alone, is exact as $Z \rightarrow \infty$. We call this functional the Magnetic ThomasFermi (MTF) functional (see Sect. IV below).

3) $Z^{4 / 3} \ll B \ll Z^{3}, Z$ large:

The magnetic field is increasingly important. To leading order all electrons will be confined to the lowest Landau band. The modified TF theory is still exact as $Z \rightarrow \infty$. In fact, the modified TF theory simplifies somewhat in this region compared to the MTF functional from the previous region. we call the new functional the Strong Thomas-Fermi (STF) functional. The only difference between STF and standard TF theory is that the usual $\rho^{5 / 3}$ is replaced by $\rho^{3} / B^{2}$, while in the MTF theory from the previous region the function that replaces $\rho^{5 / 3}$ is more complicated (see (4.1) below). The density is almost spherical and stable atoms are almost neutral. Furthermore, the atom is getting smaller. The atomic radius behaves like $Z^{1 / 5} B^{-2 / 5}=Z^{-1 / 3}\left(B / Z^{4 / 3}\right)^{-2 / 5}$. The energy behaves like $Z^{9 / 5} B^{2 / 5}=Z^{7 / 3}\left(B / Z^{4 / 3}\right)^{2 / 5}$.

4) $B \sim Z^{3}, Z$ large:

The modified TF theories are no longer applicable. Indeed, we shall in general not approximate the energy by functionals of the density $\rho$ alone. The energy is approximated by a more complicated functional to be described below in Sect. IV depending on a one particle density matrix. We call this functional the Density Matrix (DM) functional. When $B / Z^{3}$ is large enough this functional again reduces to a density functional . For the first time the atom is no longer spherical to leading order. The length scale of the atom behaves like $Z^{-1}$ and the energy like $Z^{3}$. 
5) $\quad B \gg Z^{3}, Z$ large:

In this hyper-strong case the atom is essentially one-dimensional. We can find a new functional, the Hyper-Strong (HS) functional depending only on the one-dimensional density $\bar{\rho}$ obtained from $\rho$ by integrating $\rho$ over the directions perpendicular to the field $\mathbf{B}$, i.e., $\bar{\rho}\left(x_{3}\right)=$ $\iint \rho\left(x_{1}, x_{2}, x_{3}\right) d x_{1} d x_{2}$. The energy behaves like $Z^{3}\left[\ln \left(B / Z^{3}\right)\right]^{2}$ and the length scale along the magnetic field is $Z^{-1}\left[\ln \left(B / Z^{3}\right)\right]^{-1}$, while the radius perpendicular to the field is $Z^{-1}\left(B / Z^{3}\right)^{-1 / 2}$.

The mathematically more precise statements of these results involve two energy functions $E_{\mathrm{MTF}}(N, B, Z)$ and $E_{\mathrm{DM}}(N, B, Z)$. The energy $E_{\mathrm{MTF}}(N, B, Z)$ is obtained as the minimum of the magnetic ThomasFermi functional mentioned under 2) above, and $E_{\mathrm{DM}}(N, B, Z)$ is the minimum of the density matrix functional mentioned under 4). The exact definitions of these functionals are given in Sect. IV below.

The energies $E_{\mathrm{MTF}}$ and $E_{\mathrm{DM}}$ correspond to unique minimizers for the respective functionals. We denote the densities for these minimizers by $\rho_{\mathrm{MTF}}$ and $\rho_{\mathrm{DM}}$ respectively.

In the case when $B=0$ the energy $E_{\mathrm{MTF}}(N, 0, Z)$ is the energy of standard TF theory. It is known [LS] (see also [L]) that TF theory is asymptotically exact as $Z \rightarrow \infty$ with $N / Z$ fixed, i.e.,

$$
E_{\mathrm{MTF}}(N, 0, Z) / E(N, 0, Z) \rightarrow 1 \text { as } Z \rightarrow \infty .
$$

Is the same true when $B \neq 0$ ? The answer, surprisingly, depends on the relative magnitudes of $B$ and $Z$, according to the 5 regions outlined above.

Theorem 1. Let $N / Z$ be fixed and suppose $B / Z^{3} \rightarrow 0$ as $Z \rightarrow \infty$. Then

$$
E_{\mathrm{MTF}}(N, B, Z) / E(N, B, Z) \rightarrow 1 \quad \text { as } Z \rightarrow \infty .
$$

This theorem covers the regions $1-3$ above. For the regions 4 and 5 we have

Theorem 2. Let $N / Z$ be fixed and suppose $B / Z^{4 / 3} \rightarrow \infty$ as $Z \rightarrow$ $\infty$. Then

$$
E_{\mathrm{DM}}(N, B, Z) / E(N, B, Z) \rightarrow 1 \quad \text { as } Z \rightarrow \infty .
$$

Notice that there is an overlap of the regions of validity of the two theorems. In fact, both theorems cover region 3 above. 
The energy functions satisfy the scalings

$$
E_{\mathrm{MTF}}(N, B, Z)=Z^{7 / 3} E_{\mathrm{MTF}}\left(N / Z, B / Z^{4 / 3}, 1\right)
$$

and

$$
E_{\mathrm{DM}}(N, B, Z)=Z^{3} E_{\mathrm{DM}}\left(N / Z, B / Z^{3}, 1\right)
$$

In region 2 there is a non-trivial parameter $B / Z^{4 / 3}$. Likewise in region 4 there is $B / Z^{3}$. In the other three regions these parameters enter in trivial way since they are tending either to 0 or $\infty$.

Region 1 corresponds to $B / Z^{4 / 3} \rightarrow 0$ and $B / Z^{3} \rightarrow 0$ in which case

$$
E_{\mathrm{MTF}}\left(N / Z, B / Z^{4 / 3}, 1\right) \rightarrow E_{\mathrm{MTF}}(N / Z, 0,1)
$$

which is the energy of standard TF theory.

Region 3 corresponds to $B / Z^{4 / 3} \rightarrow \infty$, in which case we have the asymptotic expansion

$$
E_{\mathrm{MTF}}\left(N / Z, B / Z^{4 / 3}, 1\right) \approx\left(B / Z^{4 / 3}\right)^{2 / 5} E_{\mathrm{STF}}(N / Z) \text { as } B / Z^{4 / 3} \rightarrow \infty
$$

where $E_{\mathrm{STF}}$ is an energy function obtained from the simplified TF theory described under 3) above.

The overlap of the regions of validity of Theorems 1 and 2 implies that

$$
E_{\mathrm{DM}}\left(N / Z, B / Z^{3}, 1\right) \approx\left(B / Z^{3}\right)^{2 / 5} E_{\mathrm{STF}}(N / Z) \text { as } B / Z^{3} \rightarrow 0
$$

Finally, region 5 corresponds to $B / Z^{3} \rightarrow \infty$, where the following asymptotic formula holds

$$
E_{\mathrm{DM}}\left(N / Z, B / Z^{3}, 1\right) \approx\left[\ln \left(B / Z^{3}\right)\right]^{2} E_{\mathrm{HS}}(N / Z) \text { as } B / Z^{3} \rightarrow \infty
$$

where $E_{\mathrm{HS}}$ is an energy function obtained from the one-dimensional functional mentioned in 5) above.

The energies $E_{\mathrm{MTF}}, E_{\mathrm{DM}}, E_{\mathrm{STF}}$ and $E_{\mathrm{HS}}$ correspond to unique minimizers for the respective functionals. We denote the densities for these minimizers by $\rho_{\mathrm{MTF}}, \rho_{\mathrm{DM}}, \rho_{\mathrm{STF}}$ and $\bar{\rho}_{\mathrm{HS}}$ respectively. We can prove that these densities approximate the quantum density $\rho$. However, to state these approximations we have to introduce different scalings in the different regions. In fact, the above approximating densities satisfy the 
following scaling relations

$$
\begin{aligned}
& \rho_{\mathrm{MTF}}(x ; N, B, Z)=Z^{2} \rho_{\mathrm{MTF}}\left(Z^{1 / 3} x ; \frac{N}{Z}, \frac{B}{Z^{4 / 3}}, 1\right) \\
& \rho_{\mathrm{STF}}(x ; N, B, Z)=Z^{2}\left(\frac{B}{Z^{4 / 3}}\right)^{6 / 5} \rho_{\mathrm{STF}}\left(\left(\frac{B}{Z^{4 / 3}}\right)^{2 / 5} Z^{1 / 3} x ; \frac{N}{Z}, 1,1\right) \\
& \rho_{\mathrm{DM}}(x ; N, B, Z)=Z^{4} \rho_{\mathrm{DM}}\left(Z x ; \frac{N}{Z}, \frac{B}{Z^{3}}, 1\right) \\
& \bar{\rho}_{\mathrm{HS}}\left(x_{3} ; N, B, Z\right)=Z^{2} \ln \left(\frac{B}{Z^{3}}\right) \bar{\rho}_{\mathrm{HS}}\left(Z \ln \left(\frac{B}{Z^{3}}\right) x_{3} ; \frac{N}{Z}, 1,1\right) .
\end{aligned}
$$

Theorem 3 (Convergence of the density). In the five different regions the following relations hold as $Z \rightarrow \infty$. These limits are all in weak $L_{\text {loc }}^{1}$ :

(1-2) If $B / Z^{4 / 3} \rightarrow \beta$, where $0 \leq \beta<\infty$ and if $N / Z=\lambda$ is fixed then

$$
Z^{-2} \rho\left(Z^{-1 / 3} x\right) \rightarrow \rho_{\mathrm{MTF}}(x ; \lambda, \beta, 1) .
$$

(3) If $B / Z^{4 / 3} \rightarrow \infty$ and $N / Z=\lambda$ is fixed then

$$
Z^{-2}\left(\frac{B}{Z^{4 / 3}}\right)^{-6 / 5} \rho\left(Z^{-1 / 3}\left(\frac{B}{Z^{4 / 3}}\right)^{-2 / 5} x\right) \rightarrow \rho_{\mathrm{STF}}(x ; \lambda, 1,1) .
$$

(4) If $B / Z^{3} \rightarrow \eta$, where $0<\eta<\infty$ and $N / Z=\lambda$ is fixed then

$$
Z^{-4} \rho_{\mathrm{DM}}\left(Z^{-1} x\right) \rightarrow \rho_{\mathrm{DM}}(x ; \lambda, \eta, 1) .
$$

(5) If $B / Z^{3} \rightarrow \infty$ and $N / Z=\lambda$ is fixed then

$$
\frac{1}{Z^{2} \ln \left(B / Z^{3}\right)} \bar{\rho}\left(\frac{x_{3}}{Z \ln \left(B / Z^{3}\right)}\right) \rightarrow \bar{\rho}_{\mathrm{HS}}\left(x_{3} ; \lambda, 1,1\right) \text {. }
$$

\section{$\S$ 3. The one-body Hamiltonian}

The spectrum of the one-body Hamiltonian $H_{\mathbf{A}}$ is described by the Landau bands $\varepsilon_{p \nu}=2 B \nu+p^{2}$, where $p$ is the momentum along the field and $\nu=0,1,2, \ldots$ is the index of the band. Owing to the spin 
degeneracy, the higher bands, $\nu \geq 1$, are twice as degenerate as the lowest band $\nu=0$.

To calculate the energy of a large, complex atom one must first study the one-body Hamiltonian $H=H_{\mathbf{A}}+V(x)$, where $V$ is an external potential. As usual, to calculate the ground state energy of a fermionic system we need to know the sum of the negative eigenvalues of the operator $H$ (with $V \leq 0$ for simplicity).

In order to estimate accurately the sum of the negative eigenvalues of $H_{\mathbf{A}}+V(x)$ we need two things: (i) a lower bound for this quantity and (ii) an asymptotic (or semiclassical) limit formula for the quantity. These are provided by Theorems 4 and 5 below. The bound (i) is needed to control errors between the true answer and the semiclassical approximation. The semiclassical limit turns out to be relevant here (after some suitable scaling) because it is equivalent to the limit $Z \rightarrow \infty$.

There is an important difference between $H_{\mathbf{A}}$ and the operator ( $\mathbf{p}-$ $\mathbf{A})^{2}$ which has no spin dependence. While the spectrum of $(\mathbf{p}-\mathbf{A})^{2}$ is $(B, \infty)$ the spectrum of $H_{\mathbf{A}}$ is $(0, \infty)$. Indeed, one can bound the sum of the negative eigenvalues of $(\mathbf{p}-\mathbf{A})^{2}-V(x)$ by $-L \int|V(x)|^{5 / 2} d x$, (where $L$ is some fixed constant) according to the standard Lieb-Thirring inequality (even with a magnetic field the proof of this inequality given in [LT] is still correct if one appeals to the diamagnetic inequality). However, in the case of $H_{\mathbf{A}}+V$ the question is somewhat more subtle. In fact, if $\int|V|^{3 / 2}<\infty$, the operator $(\mathbf{p}-\mathbf{A})^{2}+V$ has a finite number of negative eigenvalues, while the operator $H_{\mathbf{A}}+V$ can have infinitely many negative eigenvalues (compare $[\mathrm{I}]$ ). We can, however, prove [LSY] the following bound which is important in our proofs.

Theorem 1. There exist universal constants $L_{1}, L_{2}>0$ such that if we let $e_{j}(B, V), j=1,2, \ldots$ denote the negative eigenvalues of $H_{\mathbf{A}}+V$ with $V \leq 0$ then

$$
\sum_{j}\left|e_{j}(B, V)\right| \leq L_{1} B \int|V(x)|^{3 / 2} d^{3} x+L_{2} \int|V(x)|^{5 / 2} d^{3} x .
$$

We can choose $L_{1}$ as close to $2 / 3 \pi$ as we please, compensating with $L_{2}$ large.

The first term on the right side is a contribution from the lowest band, $\nu=0$. For large $B$ this is the leading term.

We now ask the question of a semiclassical analog of (3.1). Thus, consider the operator

$$
[(h \mathbf{p}-b \mathbf{a}(x)) \cdot \sigma]^{2}+v(x),
$$


where $\mathbf{a}(x)=\frac{1}{2} \hat{z} \times x, \hat{z}=(0,0,1)$ and $v \leq 0$.

If one computes the leading term in $h^{-1}$ of the sum of the negative eigenvalues of (3.2) for fixed $b$ one finds as in [HR] that there is no $b$ dependence. In our case, however, we shall not assume $b$ fixed, or more precisely not assume that $b$ is small compared with $h^{-1}$. The reason for this is that in the application to neutron stars it is not true, as we shall discuss below, that $b \ll h^{-1}$.

The interesting fact is, however, that we can prove ([LSY]) a semiclassical formula for the sum of the negative eigenvalues of the operator (3.2), which holds uniformly in $b$ (even for large $b$ ).

Theorem 5. Let $e_{j}(h, b, v), j=1,2, \ldots$, denote the negative eigenvalues of the operator (3.2), with $v \leq 0$. Then

$$
\lim _{h \rightarrow 0}\left(\sum_{j} e_{j}(h, b, v) / E_{\mathrm{scl}}(h, b, v)\right)=1,
$$

uniformly in $b$, where $E_{\mathrm{scl}}$ is the semiclassical approximation defined by

$$
\begin{aligned}
& E_{\mathrm{scl}}(h, b, v) \\
= & -\frac{1}{3 \pi^{2}} h^{-2} b \int\left(|v(x)|^{3 / 2}+2 \sum_{\nu=1}^{\infty}[|v(x)|-2 \nu b h]_{+}^{3 / 2}\right) d^{3} x .
\end{aligned}
$$

Here $[t]_{+}=t$ if $t>0$, zero otherwise.

The formula (3.3) was already implicitly noted in [Y]. The integrand in (3.3) looks peculiar, but it has the following simple physical interpretation. Take a cubic box of volume $L^{3}$ in $\mathbb{R}^{3}$ and let the number $\mu>0$ be some fixed Fermi level (or chemical potential). Then add together all the negative eigenvalues of $H_{\mathbf{A}}-\mu$. In the thermodynamic limit (large $L)$ we can do this addition simply by using the known Landau levels, and the total energy per unit volume is the integrand in (3.3) in which $|v(x)|$ is set equal to $\mu$.

For $b h \ll 1$, the right side of $(3.3)$ reduces to the standard semiclassical formula from [HR],

$$
-\frac{2}{15 \pi^{2}} h^{-3} \int|v(x)|^{5 / 2} d^{3} x
$$

(Recall that we are counting the spin which accounts for the 2 in front of the sum in (3.3).) For $b h \gg 1$, the sum in (3.3) is negligible, and we are left with the first term. 
Formula (3.3) (with $h$ replaced by 1) can be compared with the Lieb-Thirring inequality (3.1), which holds even outside the semiclassical regime. The two terms in (3.1) correspond to respectively the $b \rightarrow \infty$ (first term) and $b \rightarrow 0$ (last term) asymptotics of (3.3).

As we know from elementary thermodynamics, the energy per unit volume as a function of the particle density $(\rho(x)$ in our case) is the Legendre transform of the pressure as a function of the chemical potential $(|v(x)|)$. Thus, corresponding to $-\left(2 / 15 \pi^{2}\right)|v(x)|^{5 / 2}$ in (3.4), there is the energy $(3 / 5)\left(3 \pi^{2}\right)^{2 / 3} \rho(x)^{5 / 3}$, which is the usual kinetic energy expression in TF theory. Likewise, corresponding to (3.3) there is a kinetic energy which we call $w_{B}(\rho(x))$. It is no longer proportional to $\rho(x)^{5 / 3}$ but it is still a convex function of $\rho(x)$. It is proportional to $\rho(x)^{3} / B^{2}$ for small $\rho$, while it is asymptotically equal to $(3 / 5)\left(3 \pi^{2}\right)^{2 / 3} \rho(x)^{5 / 3}$ as $\rho(x) \rightarrow \infty$.

\section{$\S 4$. The many-electron atom}

The essential ingredient in the study of the many-electron Hamiltonian $H_{N}$ is to reduce it to a one-electron problem $H_{\mathbf{A}}+V_{\text {eff }}(x)$ with an effective mean field potential $V_{\text {eff }}(x)=-Z /|x|+\int|x-y|^{-1} \rho(y) d^{3} y$. This reduction involves approximating the repulsive energy

$$
\int\left\|\psi\left(x_{1}, \ldots, x_{N}\right)\right\|^{2} \sum_{1 \leq i<j \leq N}\left|x_{i}-x_{j}\right|^{-1} d^{3} x_{1} \ldots d^{3} x_{N},
$$

in the ground state $\psi$ by

$$
\iint \rho(x) \rho(y)|x-y|^{-1} d^{3} x d^{3} y .
$$

In standard TF theory the justification of this approximation is done by using the correlation inequality of Lieb and Oxford (see $[\mathrm{L}]$ and $[\mathrm{LO}]$ ). This very same argument (and inequality) work in the presence of a magnetic field. If $B$ is not too large compared with $Z$ it continues to be effective. However, in the hyper-strong case $B \gg Z^{3}$ the argument is no longer effective, the reason being that the correlation estimate is three dimensional in nature, while the atom is now effectively one-dimensional. The proof of a correlation estimate applicable in the hyper-strong case is difficult and will appear elsewhere ([LSY]).

The density $\rho$ appearing in the mean field potential $V_{\text {eff }}$ will not be taken to be the exact (unknown) density of the ground state, but rather an approximation to the exact density obtained from the density functionals that we shall now define. 
Armed with the foregoing, we introduce a (magnetic field dependent) $\mathrm{TF}$ theory by means of the following functional of the unknown electron density $\rho(x)$ :

$$
\begin{aligned}
\mathcal{E}_{\mathrm{MTF}}(\rho)=\int w_{B}(\rho(x)) d^{3} x & -\int Z|x|^{-1} \rho(x) d^{3} x \\
& +\frac{1}{2} \iint \rho(x)|x-y|^{-1} \rho(y) d^{3} x d^{3} y .
\end{aligned}
$$

It differs from the usual $\mathrm{TF}$ functional only in the replacement of (const.) $\rho(x)^{5 / 3}$ by $w_{B}(\rho(x))$. We call this functional the Magnetic Thomas-Fermi Functional. It is studied in detail in [LSY]. The paper $[\mathrm{TY}]$ seems to be the earliest reference that uses a Thomas-Fermi theory that takes all Landau levels into account. This theory was also studied in [FGPY] and put on a rigorous basis in [Y] for the regime $B \sim Z^{4 / 3}$.

We now choose our density $\rho$ to be the unique minimizer for $\mathcal{E}_{\mathrm{MTF}}$ constrained to the set $\int \rho \leq N$. We define the energy function that appears in Theorem 1 to be the infimum

$$
E_{\mathrm{MTF}}(N, B, Z)=\inf _{\int \rho \leq N} \mathcal{E}_{\mathrm{MTF}}(\rho) .
$$

Theorems 4 and 5 play an essential role in the proof of Theorem 1. What makes the proof work when $B \ll Z^{3}$ is the fact that in the analysis of the mean-field, one-particle Hamiltonian, $H_{\mathbf{A}}+V_{\text {eff }}(x)$, with $V_{\text {eff }}(x)=-Z /|x|+\int|x-y|^{-1} \rho(y) d^{3} y$, and with $\rho$ being the density that minimizes the TF energy, we are in the semiclassical regime. The potential $V_{\text {eff }}(x)$ has the following behavior in $Z$ and $B$

$$
\begin{aligned}
& V_{\text {eff }}(x)=Z^{4 / 3} v\left(Z^{1 / 3} x\right) \quad \text { if } B \lesssim Z^{4 / 3} \\
& V_{\text {eff }}(x)=Z^{4 / 5} B^{2 / 5} v\left(Z^{-1 / 5} B^{2 / 5} x\right) \quad \text { if } B \gtrsim Z^{4 / 3},
\end{aligned}
$$

where $v$ is a function that does not depend significantly on $B$ and $Z$.

Concentrating on the case $B \gtrsim Z^{4 / 3}$ we see, by a simple rescaling, that the Hamiltonian $H_{A}+V_{\text {eff }}(x)$ is unitarily equivalent to the operator

$$
\left.Z^{4 / 5} B^{2 / 5}[(h \mathbf{p}-b \mathbf{a}(x)) \cdot \sigma)^{2}+v(x)\right],
$$

where

$$
h=\left(B / Z^{3}\right)^{1 / 5} \quad \text { and } \quad b=\left(B^{2} / Z\right)^{1 / 5} .
$$


In the opposite case, when $B \lesssim Z^{4 / 3}$, we get $Z^{4 / 3}$ in place of $Z^{4 / 5} B^{2 / 5}$ in (4.3) and

$$
h=Z^{-1 / 3} \quad \text { and } \quad b=B / Z \text {. }
$$

When $h$ is small we can study (4.3) by semiclassical methods.

If $B \gg Z^{4 / 3}$ we can replace $w_{B}(\rho)$ by its asymptotic form and we define the Strong Thomas-Fermi functional

$$
\begin{aligned}
\mathcal{E}_{\mathrm{STF}}(\rho)=\frac{4}{3} \pi^{4} B^{-2} \int \rho(x)^{3} d^{3} x & -\int Z|x|^{-1} \rho(x) d^{3} x \\
& +\frac{1}{2} \iint \rho(x)|x-y|^{-1} \rho(y) d^{3} x d^{3} y
\end{aligned}
$$

The analysis of $E_{\mathrm{MTF}}$ and $E_{\mathrm{STF}}$, which is a separate story in itself, leads to the conclusions stated in 1),2) and 3) of Section II. Conclusions 1) and 2) were proved by Yngvason $[Y] ; 3)$ is new. Since the TF energy functional has a unique minimizing $\rho(x)$ (because $\mathcal{E}_{\mathrm{MTF}}$ is strictly convex in $\rho$ ) this $\rho$ must be spherically symmetric. Thus we are led to the following remarkable conclusion:

If $B / Z^{3} \rightarrow 0$ as $Z \rightarrow \infty$, the atom is always spherical (to leading order) despite the fact that $B$ has a leading order effect on the ground state energy.

In case $2, B \approx Z^{4 / 3}$, we cannot say that all the electrons are in the lowest Landau band, but if $B \gg Z^{4 / 3}$, they are - as the following theorem states precisely.

Theorem 6. If $\Pi_{0}^{N}$ is the projection in the physical Hilbert space onto the subspace where all electrons are in the lowest Landau band, we can define the confined energy

$$
E_{\text {conf }}(N, B, Z) \equiv \text { ground state energy of } \Pi_{0}^{N} H_{N} \Pi_{0}^{N} .
$$

Then, if $N<\lambda Z$ for some fixed $\lambda>0$, we have that

$$
\begin{aligned}
E_{\text {conf }}(N, B, Z) / E(N, B, Z) & \rightarrow 1 \\
\text { if } B \rightarrow \infty & \text { and if } Z^{4 / 3} / B \rightarrow 0 .
\end{aligned}
$$

What happens if $B \approx Z^{3}$ ? Semiclassical analysis breaks down (in the sense of being no longer asymptotically exact as $Z \rightarrow \infty$ ). The atom is no longer spherical. However, the atom is so non-semiclassical (one person called it post-modern) that another analysis becomes possible. 
This analysis, which we discuss next, is reminiscent of Hartree theory for bosons - even though it is relevant for fermionic electrons!

It is only the motion parallel to the magnetic field which can no longer be described semiclassically. The motion perpendicular to the field is still well approximated classically. To be more precise, the atom consists of a bundle of one dimensional quantum systems indexed by the position $x_{\perp}=\left(x_{1}, x_{2}\right)$ perpendicular to the field $\mathbf{B}$. The state of one of these one-dimensional systems is described by a finite family of orthogonal functions $e_{x_{\perp}}^{(j)}, j=1,2 \ldots$ in $L^{2}(\mathbf{R})$ which are not normalized but satisfy $\left\|e_{x_{\perp}}^{(j)}\right\| \leq B / 2 \pi$. This condition follows from the Pauli principle and the fact that the two-dimensional density of states in the lowest Landau band is exactly $B / 2 \pi$.

We can combine the functions $e_{x_{\perp}}^{(j)}, j=1,2, \ldots$ into a density matrix

$$
\gamma: x_{\perp} \mapsto \gamma_{x_{\perp}}\left(x_{3}, y_{3}\right)=\sum_{j} e_{x_{\perp}}^{(j)}\left(x_{3}\right) \overline{e_{x_{\perp}}^{(j)}\left(y_{3}\right)}
$$

Then $\gamma$ satisfies

(a) $0 \leq \gamma_{x_{\perp}} \leq(B / 2 \pi) I$ as an operator on $L^{2}(\mathbf{R})$

(b) $\int_{\mathbf{R}^{2}} \operatorname{Tr}_{L^{2}(\mathbf{R})}\left[\gamma_{x_{\perp}}\right] d^{2} x_{\perp}=N=$ the total number of electrons.

We can now approximate the energy by the functional

$$
\begin{aligned}
\mathcal{E}_{\mathrm{DM}}(\gamma)= & \int_{\mathbf{R}^{2}} \operatorname{Tr}_{L^{2}(\mathbf{R})}\left[\left(-\partial_{3}^{2}-Z|x|^{-1}\right) \gamma_{x_{\perp}}\right] d^{2} x_{\perp} \\
& +\frac{1}{2} \iint \rho_{\gamma}(x) \rho_{\gamma}(y)|x-y|^{-1} d^{3} x d^{3} y,
\end{aligned}
$$

where $\rho_{\gamma}(x)=\gamma_{x_{\perp}}\left(x_{3}, x_{3}\right)$.

We denote

$$
E_{\mathrm{DM}}(N, B, Z)=\inf \{\mathcal{E}(\gamma): \gamma \text { satisfies (a) and (b) above }\} .
$$

This is the function appearing in Theorem 2. The Pauli principle comes into play in this theory only in condition (a). The proof of Theorem 2 is straightforward as soon as one has made the reduction to a one body problem and realized that condition (a) follows from the confinement to the lowest Landau band.

The Euler-Lagrange equation for the $\mathcal{E}_{\mathrm{DM}}$ minimization problem implies that the functions $e_{x_{\perp}}^{(j)}$ are eigenfunctions of the one-dimensional Schrödinger operator $h_{x_{\perp}}=-\frac{d^{2}}{d x_{3}^{2}}-V_{\text {eff }}(x)$ where, as before, the effective potential is $V_{\text {eff }}(x)=-Z /|x|+\int|x-y|^{-1} \rho_{\gamma}(y) d^{3} y$ with $\rho_{\gamma}$ being the density corresponding to the minimizer $\gamma$ for $\mathcal{E}_{\mathrm{DM}}$ 


\section{$\S 5$. The super strong case $B \gg Z^{3}$}

We shall present here the correct energy functional of the density when $B \gg Z^{3}$, and very briefly indicate what is involved in proving the correctness of the approximation.

The first step is to show that when $B / Z^{3}$ is larger than some critical value then the minimizing $\gamma$ for $\mathcal{E}_{\mathrm{DM}}$ is rank one for every $x_{\perp}$. Since the eigenfunction of $\gamma_{x_{\perp}}$ must be the ground state of $h_{x_{\perp}}$ we can conclude that it is a positive function. In this case we can write $\gamma_{x_{\perp}}\left(x_{3}, y_{3}\right)=$ $\sqrt{\rho\left(x_{\perp}, x_{3}\right)} \sqrt{\rho\left(x_{\perp}, y_{3}\right)}$ where $\rho(x)=\rho_{\gamma}(x)$.

The functional $\mathcal{E}_{\mathrm{DM}}$ thus becomes a density functional when $B / Z^{3}$ is large enough.

$$
\begin{aligned}
\mathcal{E}_{\mathrm{DM}}(\gamma)=\mathcal{E}_{\mathrm{SS}}(\rho)=\int & \left(\frac{\partial}{\partial x_{3}} \sqrt{\rho(x)}\right)^{2} d^{3} x-\int \frac{Z}{|x|} \rho(x) d^{3} x \\
& +\frac{1}{2} \int \rho(x)|x-y|^{-1} \rho(y) d^{3} x d^{3} y
\end{aligned}
$$

with the condition that

$$
\int \rho\left(x_{1}, x_{2}, x_{3}\right) d x_{3} \leq \frac{B}{2 \pi} \text { for all }\left(x_{1}, x_{2}\right) .
$$

Then

$$
\begin{aligned}
E_{\mathrm{DM}}(N, B, Z) & \left.=E_{\mathrm{SS}}(N, B, Z)\right) \\
& =\inf \left\{\mathcal{E}_{\mathrm{SS}}(\rho): \int \rho \leq N, \rho \text { satisfies }(5.2)\right\} .
\end{aligned}
$$

We can now ask for the limit of $\mathcal{E}_{\mathrm{SS}}$ if $B / Z^{3} \rightarrow \infty, Z \rightarrow \infty$ and $N / Z$ is fixed. With some effort one can prove that $\mathcal{E}_{\mathrm{SS}}$ then simplifies to another functional, which we call the hyper-strong functional of a one-dimensional density $\rho_{1}(x), x \in \mathbb{R}$. That is, the atom is now so thin compared to its length that only the average density and its variation along the direction parallel to $\mathbf{B}$ matter.

It is convenient, in defining this average density, to rescale the variables. Thus, setting $\eta \equiv B /\left(2 \pi Z^{3}\right)$, and taking $(Z \ln \eta)^{-1}$ as the unit of length, we define

$$
\begin{aligned}
\rho_{1}(x) & \equiv \frac{1}{Z^{2} \ln \eta} \bar{\rho}\left(\frac{1}{Z \ln \eta} x\right) \\
& \equiv \frac{1}{Z^{2} \ln \eta} \int \rho\left(x_{1}, x_{2}, \frac{1}{Z \ln \eta} x\right) d x_{1} d x_{2}
\end{aligned}
$$


which has the normalization $\int \rho_{1}(x) d x=N / Z$. The hyper-strong functional is

$$
\mathcal{E}_{\mathrm{HS}}\left(\rho_{1}\right)=\int_{-\infty}^{\infty}\left(\frac{d}{d x} \sqrt{\rho_{1}(x)}\right)^{2} d x-\rho_{1}(0)+\frac{1}{2} \int_{-\infty}^{\infty} \rho_{1}(x)^{2} d x
$$

In other words, apart from some scalings, the Coulomb potential is replaced by a Dirac delta function! Using (5.5) we define a rescaled energy

$$
E_{\mathrm{HS}}(N / Z) \equiv \inf _{\int \rho_{1}=N / Z} \mathcal{E}_{\mathrm{HS}}\left(\rho_{1}\right) .
$$

We assert that under the conditions stated above, $Z^{3}(\ln \eta)^{2} E_{\mathrm{HS}}(N / Z)$ $/ E(N, B, Z) \rightarrow 1$ as $Z \rightarrow \infty, B / Z^{3} \rightarrow \infty$ and $N / Z$ is fixed.

A remarkable fact is that the minimizing $\rho_{1}$ can be evaluated exactly. The Euler-Lagrange equation is (with $\psi^{2} \equiv \rho_{1}$ and Lagrange multiplier $\mu)$

$$
-\ddot{\psi}(x)-\psi(0) \delta(x)+\psi^{3}(x)=-\mu \psi(x) .
$$

With $\lambda \equiv N / Z$, there are solutions only for $\lambda \leq 2$ (not $\lambda \leq 1$ as in TF theory):

$$
\psi(x)=\frac{\sqrt{2}(2-\lambda)}{2 \sinh \left[\frac{1}{4}(2-\lambda)|x|+c\right]} \quad \text { for } \lambda<2
$$

$$
\psi(x)=\sqrt{2}(2+|x|)^{-1} \quad \text { for } \lambda=2,
$$

with $\tanh c=(2-\lambda) / 2$. The energy is

$$
E_{\mathrm{HS}}(\lambda)=\mathcal{E}_{\mathrm{HS}}\left(\psi^{2}\right)=-\frac{1}{4} \lambda+\frac{1}{8} \lambda^{2}-\frac{1}{48} \lambda^{3} .
$$

\section{References}

[FGP] Fushiki, I., Gudmundsson, E.H. and Pethick, C.J., Surface structure of neutron stars with high magnetic fields, Astrophys. Jour., 342 (1989), 958-975.

[FGPY] Fushiki, I., Gudmundsson, E.H., Pethick, C.J. and Yngvason, J., Matter in a magnetic field in the Thomas-Fermi and related theories, Ann. of Phys. (NY), 216 (1992), 29-72. 
[HR] Helffer, B. and Robert, D., Calcul fonctionel par la transformeé de Mellin et operateurs admissible, Jour. Func. Anal., 53 (1983), 246-268.

[I] Ivrii, V., in preparation.

[K] Kadomtsev, B. B., Heavy Atoms in an Ultrastrong Magnetic Field, Soviet Phys. JETP, 31 (1970), 945-947.

[L] Lieb, E.H., Thomas-Fermi and related theories of atoms and molecules, Rev. Mod. Phys., 54 (1981). Errata, 55, 311 (1982)

[LS] Lieb, E.H. and Simon, B., The Thomas-Fermi theory of atoms, molecules and solids,, Adv. in Math., 23 (1977), 22-116.

[LSY] Lieb, E.H., Solovej, J.P. and Yngvason, J., Heavy Atoms in the Strong Magnetic Field of a Neutron Star, Phys. Rev. Lett., 69, 749-752 (1992). This is an announcement. The details will appear in two parts: Asymptotics of Heavy Atoms in High Magnetic Fields: I. Lowest Landau Band Regions (Commun. Pure Appl. Math., to appear in the issue dedicated to H. P. McKean, 1994) and II. Semiclassical Regions (in preparation). The first paper covers regions III, IV, V; the second covers I, II, III.

[LT] Lieb, E.H. and Thirring, W.E., A bound for the moments of the eigenvalues of the Schrödinger Hamiltonian and their relation to Sobolev inequalities, Studies in Mathematical Physics: Essays in Honor of Valentine Bargmann, ed. E.H. Lieb, B. Simon and A.S. Wightman, Princeton University Press (1977).

[MRS] Mueller, R. O., Rau, A.R.P. and Spruch, L., Statistical model of atoms in intense magnetic fields, Phys. Rev. Lett., 26 (1971), p. 1136.

[R] Ruderman, M., Matter in superstrong magnetic fields: The surface of a neutron star, Phys. Rev. Lett., 27 (1971), 1306-1308.

[TY] Tomishima, Y and Yonei, K., Thomas Fermi theory for atoms in a strong magnetic field, Progr. Theor. Phys., 59 (1978), 683-696.

[Y] Yngvason, J., Thomas-Fermi theory for matter in a magnetic field as a limit of quantum mechanics, Lett. Math. Phys., 22 (1991), 107-117.

Departments of Mathematics and Physics

Princeton University

Box 708

Princeton, NJ 08544

U. S. A. 